\title{
Multiple Coexisting Attractors and Hysteresis in the Generalized Ueda Oscillator
}

\author{
Kehui Sun, ${ }^{1,2}$ A Di-li Duo Li-kun, ${ }^{1}$ Yanqing Dong, ${ }^{1}$ Huihai Wang, ${ }^{2}$ and Ke Zhong ${ }^{2}$ \\ ${ }^{1}$ School of Physics Science and Technology, Xinjiang University, Urumqi 830046, China \\ ${ }^{2}$ School of Physics and Electronics, Central South University, Changsha 410083, China \\ Correspondence should be addressed to Kehui Sun; kehui@csu.edu.cn
}

Received 27 August 2013; Accepted 11 November 2013

Academic Editor: Hai Yu

Copyright (c) 2013 Kehui Sun et al. This is an open access article distributed under the Creative Commons Attribution License, which permits unrestricted use, distribution, and reproduction in any medium, provided the original work is properly cited.

A periodically forced nonlinear oscillator called the generalized Ueda oscillator is proposed. The restoring force term of this equation consists of a nonlinear function $\operatorname{sgn}(x)$ and an absolute function with a variant power. Dynamics is investigated by detailed numerical analysis as well as dynamic simulation, including the largest Lyapunov exponent, phase diagrams, and bifurcation diagrams. Multiple coexisting attractors and complex hysteresis phenomenon are observed. The results show that this system has rich dynamical behaviors, and it has a promising application in the fields of science and engineering.

\section{Introduction}

It is well known that the periodically forced nonlinear oscillator is one of the earliest classes to generate chaos. Generally, a forced chaotic oscillator has the form $\ddot{x}+f(\dot{x}, x)=$ $F \sin \omega t$, where the function $f(\dot{x}, x)$ contains at least one nonlinearity in the damping $(\dot{x})$ term or the restoring force $(x)$ term. For the damping term, the examples include the van der Pol equation [1] and the Rayleigh differential equation [2]. For the restoring force term, the examples include the Duffing oscillator [3], the Ueda oscillator [4], Duffing's twowell oscillator [5], and damped-driven Duffing oscillator [6]. Several hybrid chaotic forced oscillators have been studied by combining the two cases above, such as the Rayleigh-Duffing oscillator [7] and the Duffing-van der Pol oscillator [8]. Some systems in which $f(\dot{x}, x)$ is a more complicated nonlinear function have been studied [9]. Here, we are interested in the case that the nonlinearity is the combination of signum function and absolute value function in the restoring force term. On one hand, the signum function describes a class of simple discontinuous switching structures, which can be simply structured and easily designed with cost-effective electronic circuit realizations or one type of the most important nonsmooth structures, which may easily create complex phenomena [10]. On the other hand, the absolute value function is a simply continuous piecewise-linear function which is usually applied in the forced systems to generate chaos [11-13]. So it has important significance to investigate this class of nonlinear system. Interestingly, we also find multiple attractors coexisting and hysteresis phenomenon in this oscillator. Some of dynamical systems are characterized by the coexistence of more than two attractors in some regions of parameter space [14]. Multiple attractors coexisting as a typical bifurcation lead to unpredictable behavior of trajectories and are considered as a source of unpredictability of a nonlinear system [15]. At the same time, hysteresis also is a typical nonlinear phenomenon and is encountered in many scientific fields, including magnetism, superconductor, granular media, and population dynamics. Some characteristic examples exhibit hysteresis, such as one-dimensional map model [16], the Van der Pol oscillator [17], and SDOF oscillator [18].

In this letter, we investigate the dynamics of the generalized Ueda oscillator in which the restoring force term is replaced by the nonlinear function $\operatorname{sgn}(x)|x|^{\alpha}$. The power $\alpha$ of the absolute is a variable parameter for representing a more general function. This paper is organized as follows. In Section 2, we present the forced oscillator model and its attractors. In Section 3, the dynamics of this system is analyzed by numerical simulations including bifurcations and the routes to chaos, multiple coexisting attractors, and 


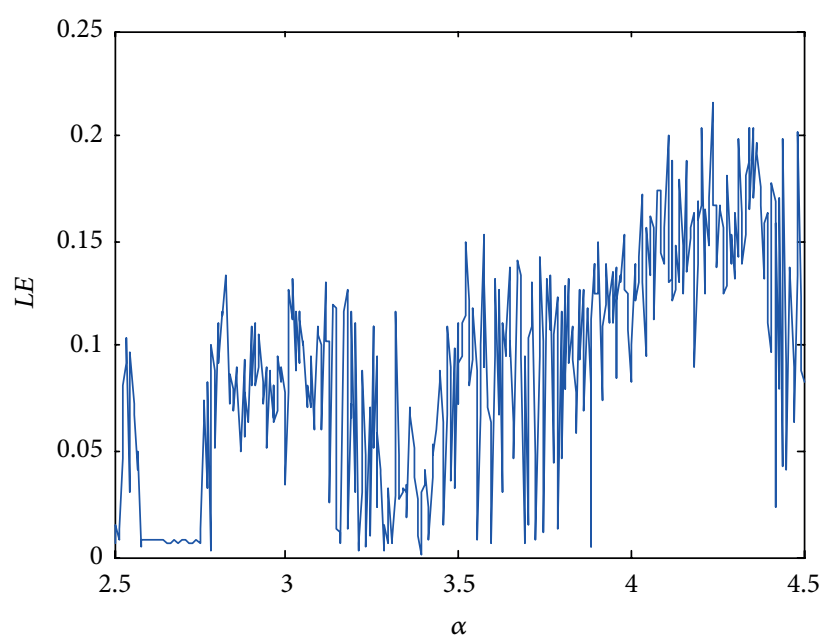

Figure 1: Maximum $L E$ of system (1) with $b=0.05, F=7.5$, and initial condition $(0.9069,0.2445,0.1)$.

hysteresis and transient chaos. Finally, we summarize the results and indicate future directions.

\section{Generalized Ueda Oscillator}

Consider the following nonautonomous differential equation:

$$
\ddot{x}+b \dot{x}+\operatorname{sgn}(x)|x|^{\alpha}=F \sin (\omega t),
$$

where, $b, \alpha, F, \omega$ are positive constants and $\operatorname{sgn}(x)$ is the signum function which is either +1 or -1 depending on whether its argument $x$ is positive or negative, respectively. For simplicity, we will set the parameter $\omega=1$ as described in [19] and consider $b, \alpha$, and $F$ as the control parameters. Obviously, this equation represents a class of general systems. For example, if $b=0$ and $0.5 \leq \alpha \leq 11$, system (1) becomes the so-called "simplest sinusoidally forced chaotic system" studied in [20]. If $b=0.05, \alpha=3$ and $F=7.5$, system (1) becomes the Ueda oscillator proposed by Ueda [21]. So system (1) is called the generalized Ueda oscillator. Noticing that the periodically forced term $F=\sin (\omega t)$ is a function with time, nonautonomous system (1) can be changed to a three-dimensional autonomous system

$$
\begin{aligned}
& \dot{x}=y, \\
& \dot{y}=-b y-\operatorname{sgn}(x)|x|^{\alpha}+F \sin (z), \\
& \dot{z}=\omega .
\end{aligned}
$$

\section{Dynamical Behaviors of the System}

3.1. Calculating the Largest Lyapunov Exponent. Lyapunov exponents are the best indicators to categorize the different classes of nonlinear phenomena. A positive Lyapunov exponent confirms chaos. If a system equation is given, algorithms in [22] can be applied to calculate the largest or all of the exponents. Alternatively, the method of embedded dimensions may be applied to time series resulting from simulations and experiments to estimate the Lyapunov exponent [23]. These approaches only work satisfactorily for smooth systems, that is, those where the vector field is continuously differentiable. For example, if parameters are $a=1.4, b=1$, $F=1.1, \omega=1$, then system (1) is a limit cycle, while the largest Lyapunov exponent is positive. Obviously, it is incorrect, which was named a chaotic limit cycle paradox in [24]. To calculate the Largest Lyapunov exponent of system (1) correctly, the signum function should be replaced by continuous hyperbolic tangent function [25]:

$$
\operatorname{sgn}(x) \longrightarrow \tanh (n x)
$$

where $n$ is a constant. The question is how to determine the range of $n$. If $n$ is a very small number, then hyperbolic tangent function is far away from signum function. If $n$ is a very big number, it is nearly equal to signum function. The results are incorrect in both of the cases. So $n$ should be chosen from $2^{4}$ to $2^{12}$ according to [26]. The largest Lyapunov exponent is calculated with different control parameter $\alpha$ as shown in Figure 1. Obviously, system (1) is chaotic within the range of $(2.5,4.5)$ with some small periodic windows.

3.2. Bifurcations and Routes to Chaos. As we know, a bifurcation diagram provides a global picture of different types of motions existed in association with the bifurcation parameter. The bifurcation diagrams for the range of parameter $\alpha \in$ $[2,5]$ with $b=0.2, F=7.5$, and different initial conditions are presented in Figure 2. It shows that periodic motion and chaotic motion coexist for the range of $\alpha \in[3.947,4.320]$. The parameter $\alpha$ in Figures 2(a) and 2(c) increases from 2 to 5 (forward tracking), while the parameter $\alpha$ in Figures 2(b) and 2(d) decreases from 5 to 2 (backward tracking). Obviously, the bifurcation diagrams are different, and it indicates that there exists the hysteretic phenomenon in this system, which will be discussed in Section 3.4 .

Now let $\alpha=4, F=7.5$, and vary the parameter $b$ from 0.01 to 0.7 . The initial states of the forced system are 


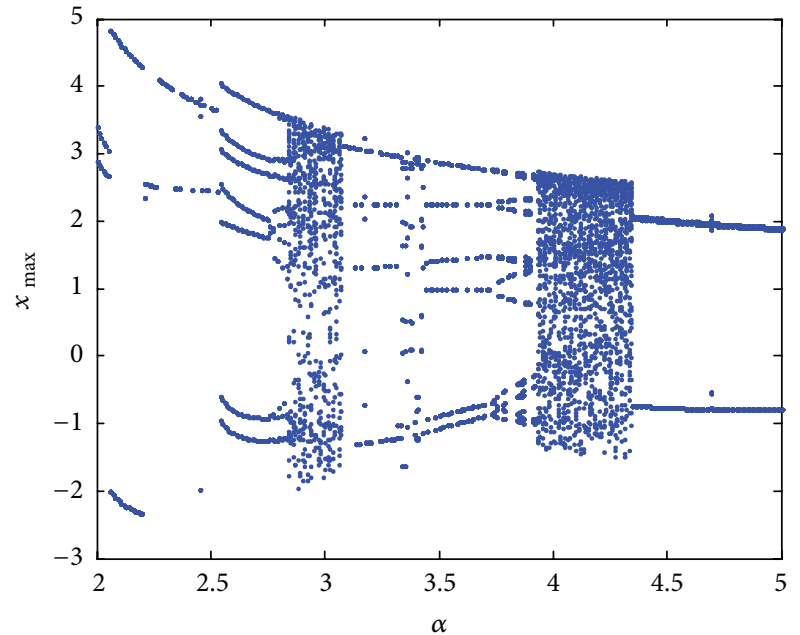

(a)

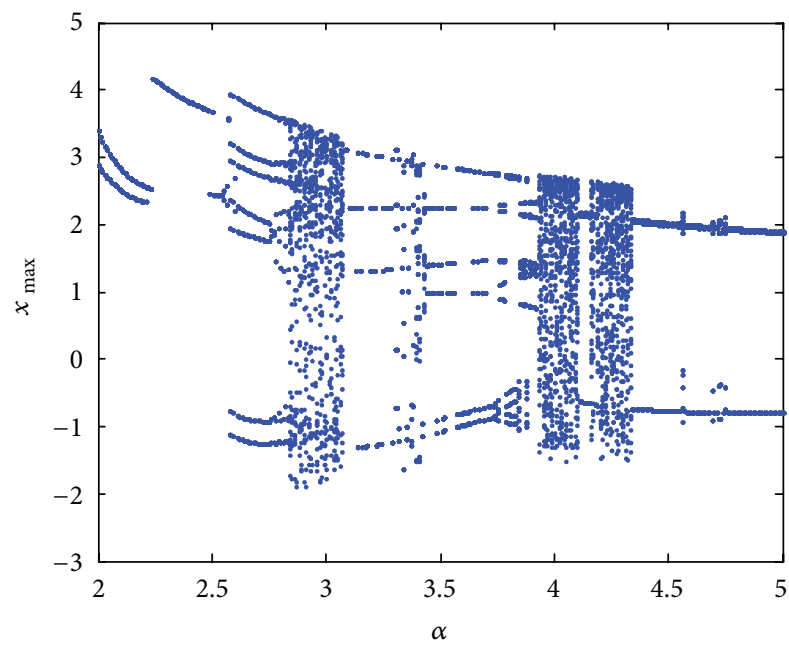

(c)

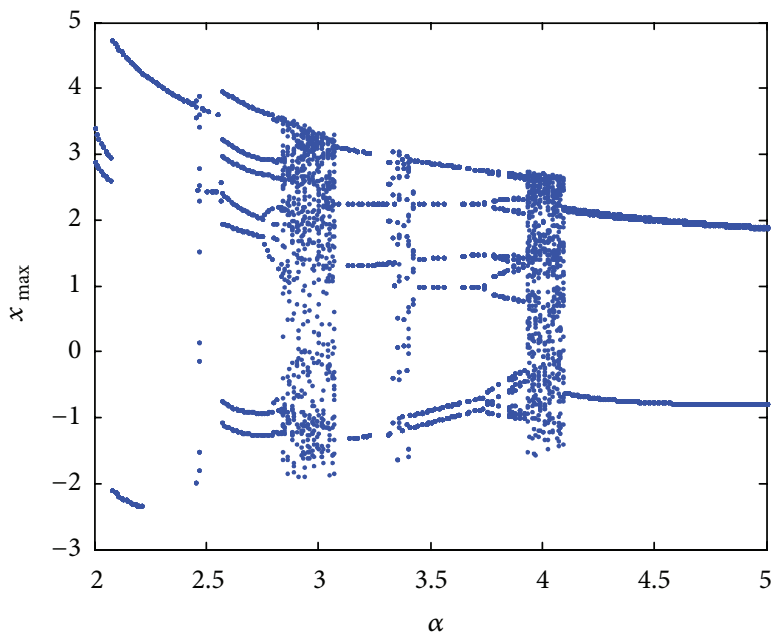

(b)

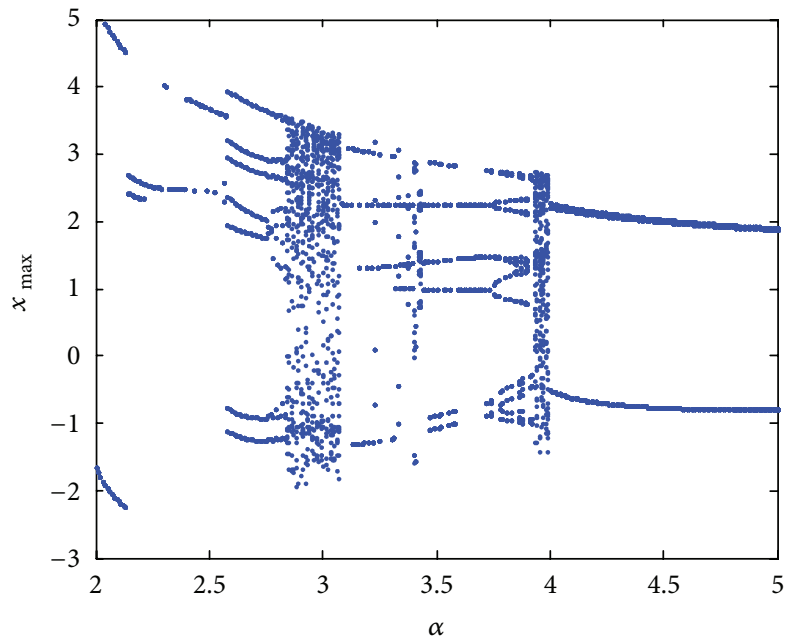

(d)

FIGURE 2: Bifurcation diagrams for parameter $\alpha$ with different initial conditions (a) IC: $(2.5,0,0)$; (b) IC: $(-0.9859,-2.6871,499.9980)$; (c) IC: $(2.8263,-2.1674,999.9960)$; (d) IC: $(-0.8689,1.0182,1.5000 e+003)$.

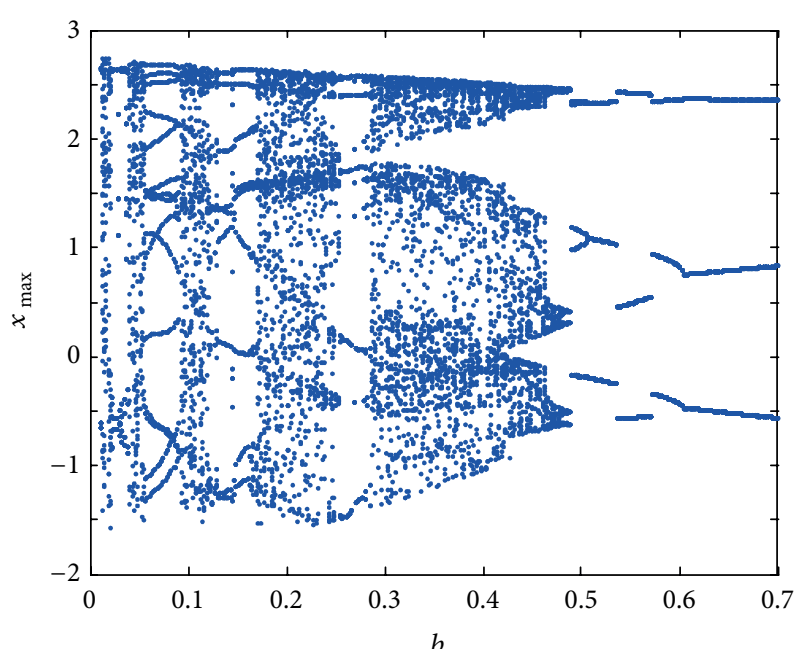

FIGURE 3: Bifurcation diagram for parameter $b$ with $\alpha=4, F=7.5$, and initial condition $[2.5,0,0]$.

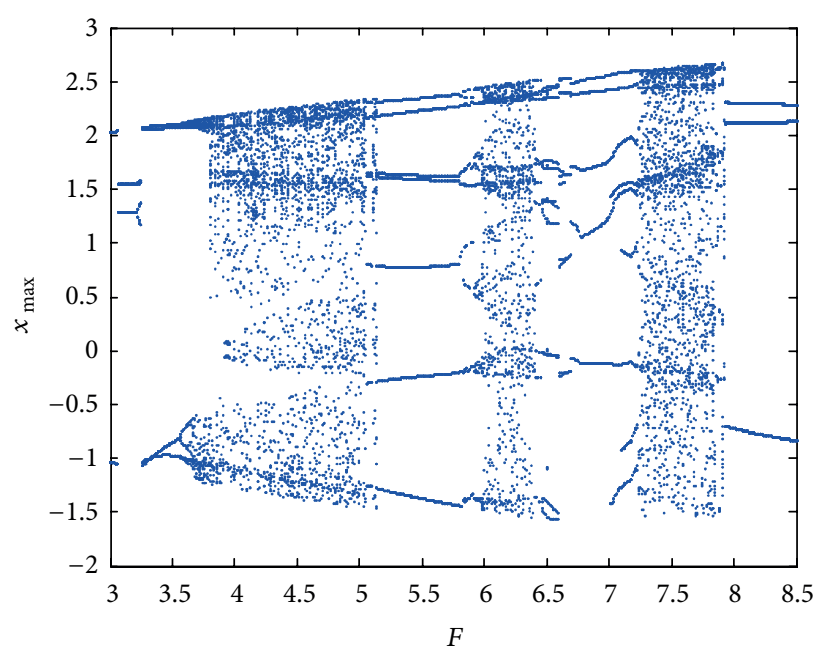

FIGURE 4: Bifurcation diagram for parameter $F$ with $\alpha=4, b=0.2$, and initial condition $[2.5,0,0]$. 


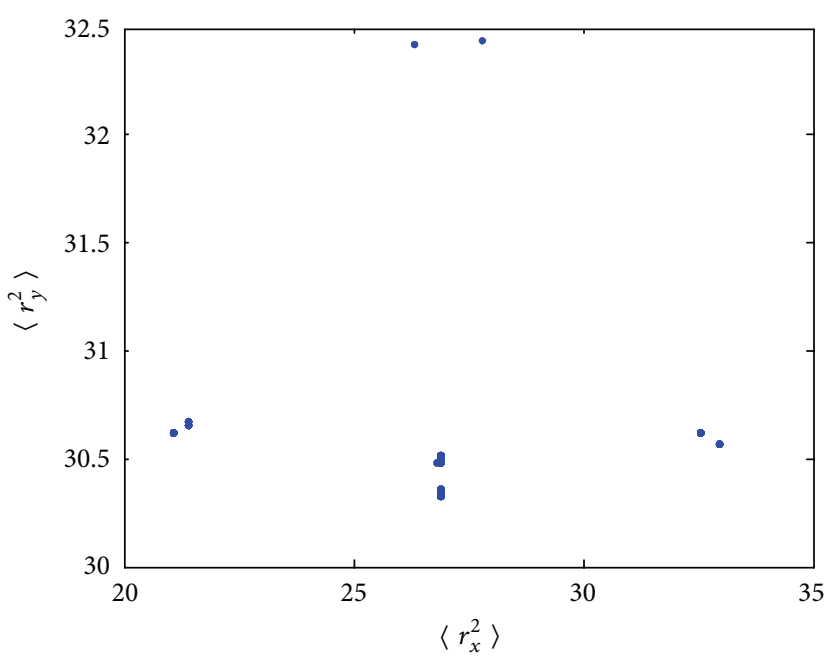

(a)

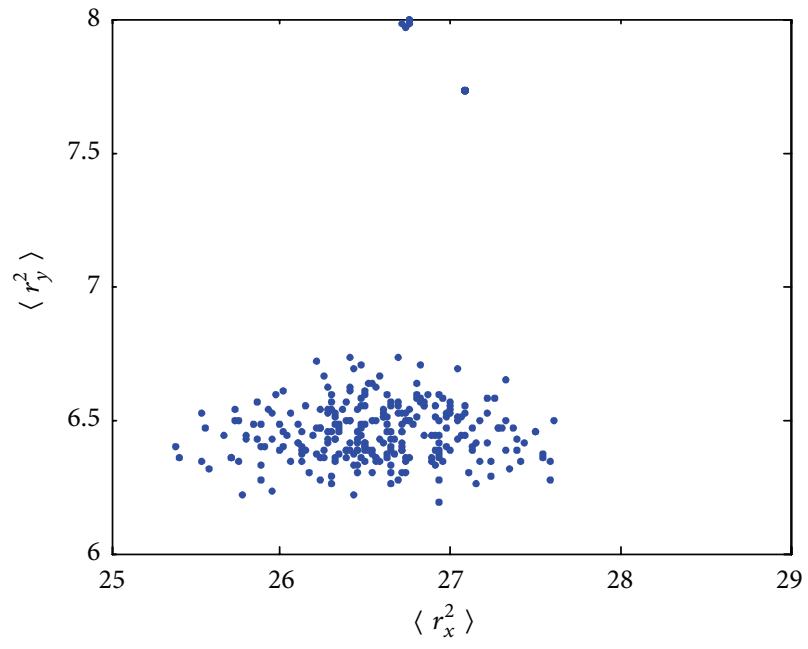

(b)

Figure 5: Multiple coexisting attractors in system (1) with different parameter $\alpha$ (a) $\alpha=3.4$, (b) $\alpha=4$.

$x(0)=2.5, y(0)=0$, and $z(0)=0$. The step size for parameter $b$ is 0.002 , and then the bifurcation diagram was obtained as shown in Figure 3, which indicates that the system is chaotic with several periodic widows at the range $b \in(0,0.5)$. When the parameter $b$ is decreased from 0.7 , the system enters into chaos by a period-doubling bifurcation. During the process of evolution, it undergoes interior crisis, pitchfork bifurcation, and tangent bifurcation.

Now let $\alpha=4, b=0.2$, and vary the parameter $F$ from 3 to 8.5. The initial states of the forced system are $x(0)=2.5$, $y(0)=0$, and $z(0)=0$. The step size for $F$ is 0.01 , and the resulting bifurcation diagram is shown in Figure 4. It shows that the system is chaotic with at least two windows at the range $F \in(3,8.5)$. When the parameter $F$ is increased from 3 , the system enters into chaos by a period-doubling bifurcation. When the parameter $F$ is decreased from 8.5, the system enters into chaos by a boundary crisis. During the process of evolution, it also experiences interior crisis, pitchfork bifurcation, and tangent bifurcation.

3.3. Multiple Attractor Bifurcations. The system described here provides an opportunity to study bifurcations of multiple attractors. For this purpose, the single scalar definition of an attractor proposed in [27] is applied as follows:

$$
\begin{aligned}
& \left\langle r_{x}^{2}\right\rangle=\lim _{N \rightarrow \infty} \frac{1}{N} \sum_{i=1}^{N}\left(x_{i}-x_{\text {ref }}\right)^{2}, \\
& \left\langle r_{y}^{2}\right\rangle=\lim _{N \rightarrow \infty} \frac{1}{N} \sum_{i=1}^{N}\left(y_{i}-y_{\text {ref }}\right)^{2},
\end{aligned}
$$

which are the mean square deviations of the attractor from the reference point $\left(x_{\text {ref }}, y_{\text {ref }}\right)$ projected onto $x, y$-axis of $x-y$ plane, respectively. Appropriate choice of $x_{\text {ref }}$ and $y_{\text {ref }}$ will obtain a unique and different value of $\left\langle r_{x}^{2}\right\rangle$ and $\left\langle r_{y}^{2}\right\rangle$ for each attractor. For the fixed parameters and many different initial conditions chosen randomly, multiple coexisting attractors will be indicated by values of $\left\langle r_{x}^{2}\right\rangle$ or $\left\langle r_{y}^{2}\right\rangle$ that cluster around distinct values. Abrupt change in the value or slope of $\left\langle r_{x}^{2}\right\rangle$ or $\left\langle r_{y}^{2}\right\rangle$ as a parameter will indicate a discontinuous (catastrophic or subcritical) or continuous (subtle or supercritical) bifurcation, respectively. Here, the reference point is taken as $(5,5)$ and initial conditions are chosen from a normal random distribution with mean 0 and variance 1.0, although other choices give similar results.

Figure 5 shows that multiple attractors coexist in system (1) with $b=0.05, F=7.5$, and different parameter $\alpha$. Obviously, there exist 8 attractors in system (1) with $\alpha=3.4$ as shown in Figure 5(a), and all the attractors are limit cycles with different period. But only three attractors coexist in system (1) with $\alpha=4$ as shown in Figure 5(b), and it indicates that there are two limit cycles with different period and one chaotic attractor in this case. The coexisting periodic attractor is presented symmetrically as shown in Figure 6.

3.4. Hysteresis and Transient Chaos. In this system, as the parameter $\alpha$ varies, a complex hysteresis phenomenon is observed as illustrated in Figure 7. As $\alpha$ increases from 3 , the evolution of the system starts from periodic state, then it enters chaos by period-doubling at $\alpha=3.93$. It is followed by several periodic windows, chaotic attractors again, and a jump back onto the periodic solution at $\alpha=4.32$. However, when we start to decrease from $\alpha=5$, it undergoes another evolution route, which undergoes low period, then low period or high period, chaos, and high period. There is a multistate coexisting area at the range $\alpha \in[3.947,4.32]$. It means that the dynamical behaviors at this area consist of three branches, including chaos, high period, and low period as shown in Figure 8, which is more complex than that which was presented in [16].

In addition to coexisting attractors and complex hysteresis, a chaotic transient is observed, which seems chaotic during some transient period, but finally falls into a periodic 


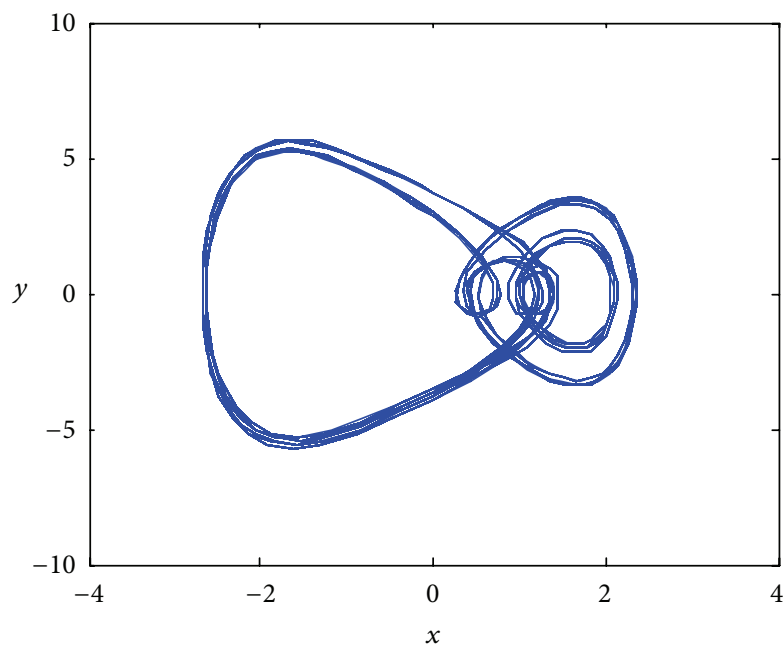

(a)

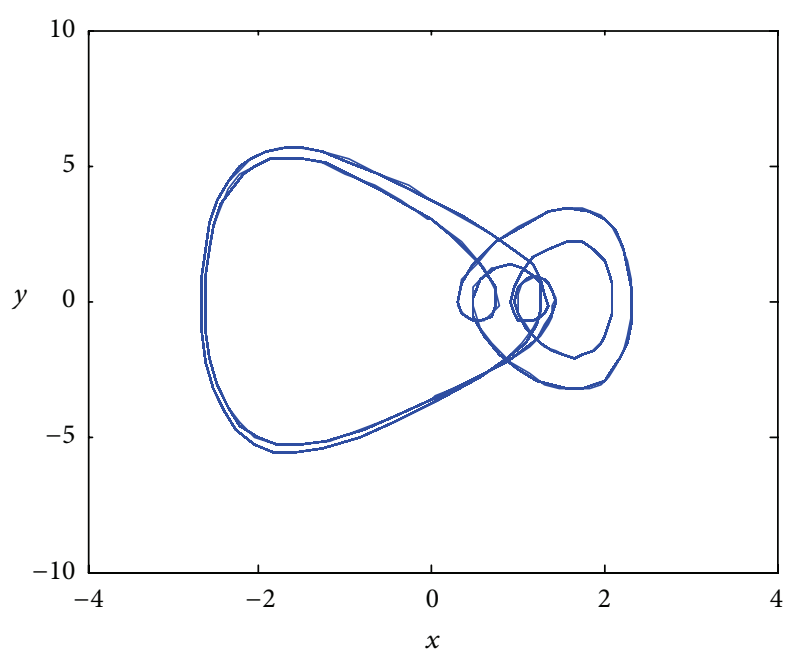

(c)

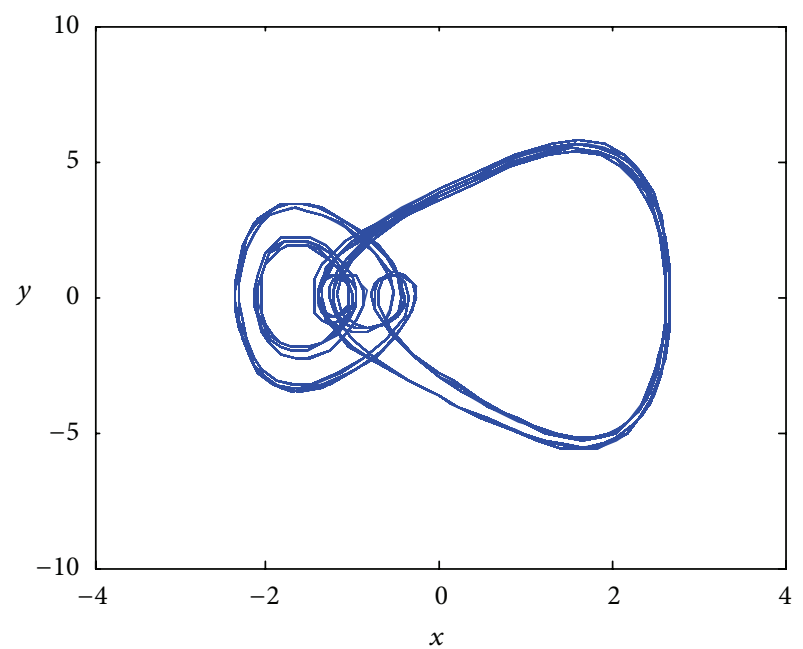

(b)

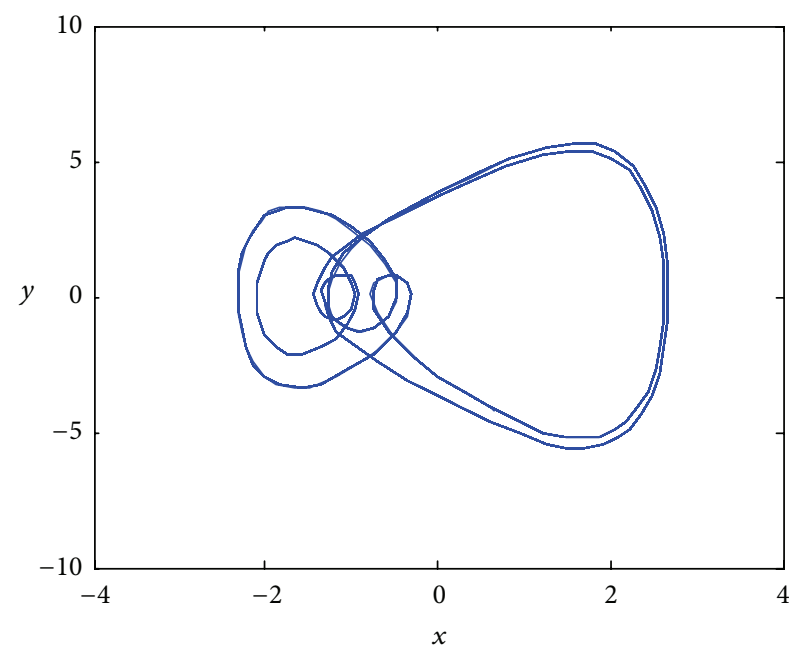

(d)

FIGURE 6: Multiattractor coexisting with $\alpha=3.91$ (a) IC: $(0.8538,1.2832,3000)$; (b) IC: $(-1.3452,-0.4867,3000)$; (c) IC: (1.5145, -2.0286 , 2000); (d) IC: $(0.6565,-4.2041,2000)$.

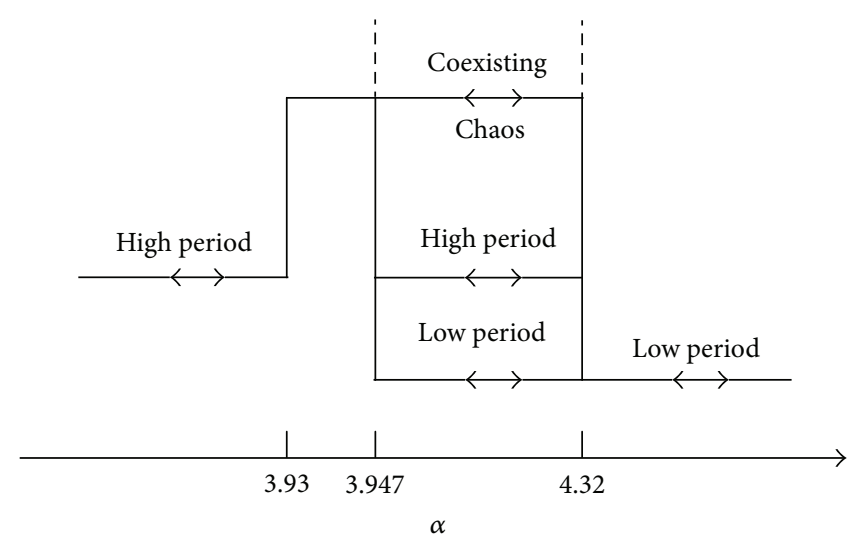

FIgURE 7: Hysteresis in system (1) with varying $\alpha$. 


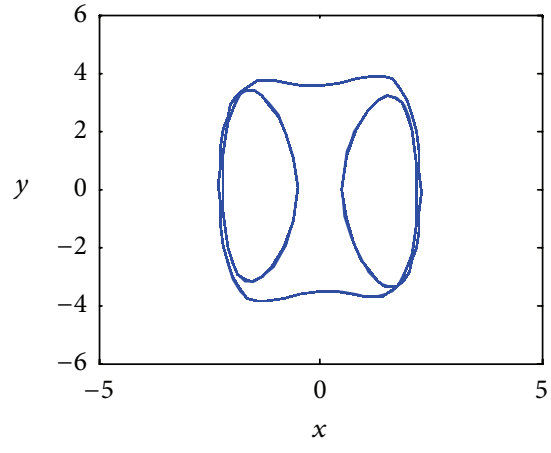

(a)

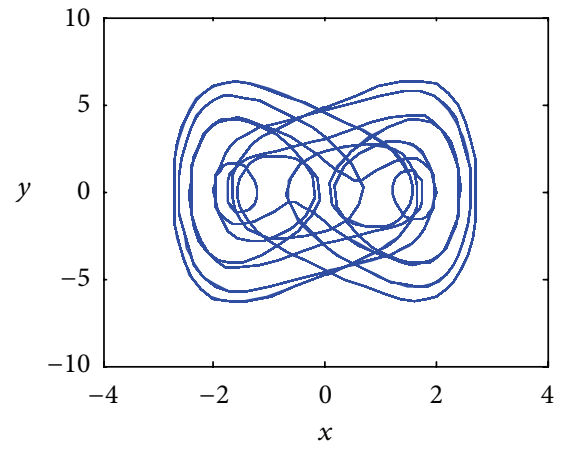

(b)

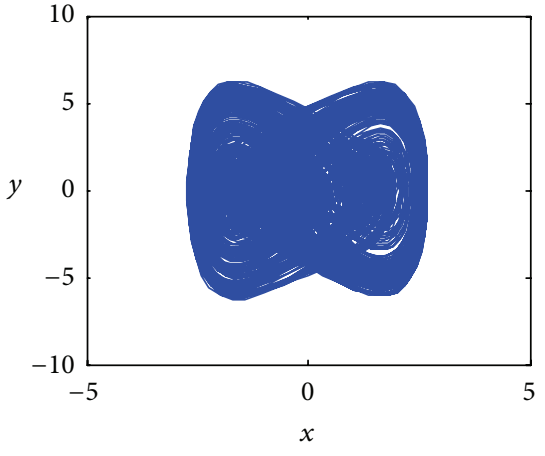

(c)

Figure 8: Multiattractor coexisting with $\alpha=4$ and different initial conditions (a) low period (IC: 1.9189, -3.092, 1000); (b) high period (IC: $1.9726,5.4330,1000)$; (c) chaos (IC: $0.6921,1.3179,0)$.

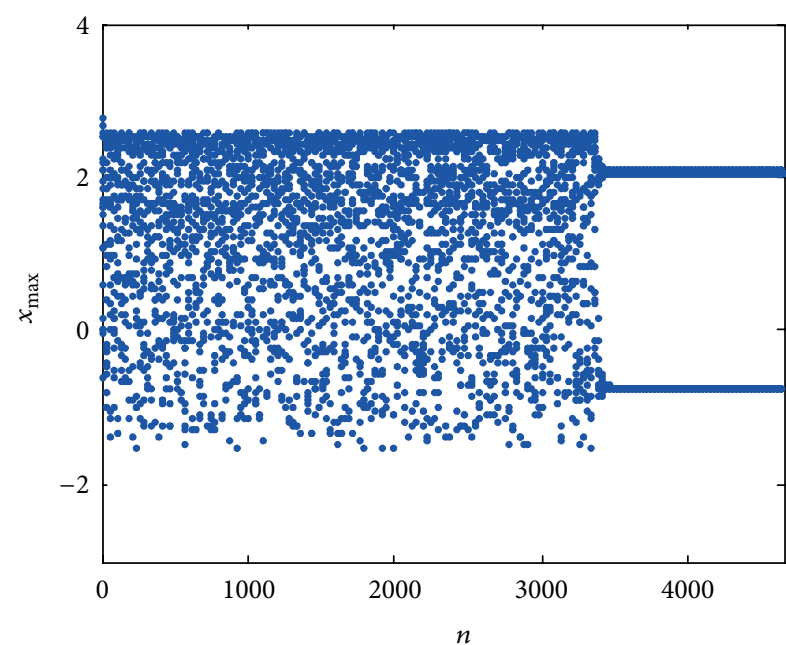

FIGURE 9: Chaotic transient at $\alpha=4.34$.

attractor. This chaotic transient phenomenon has been mentioned for the standard Lorenz system [28], and superlong chaotic transients (referred to as "supertransients") have been observed in chaotic circuit experiments [29]. For the forced nonlinear oscillator, orbits, which turn out to be periodic, are preceded by chaotic transient which lasts about 3500 driver periods. Figure 9 shows the evolution of one coordinate of an orbit which is first caught up in a chaotic transient for about 3500 periods in the case of $a=4.34$ with initial condition $\left[x_{0}, y_{0}, z_{0}\right]=[2.5,0,0]$ and then settles into limit cycle. The transient is apparently chaotic with the largest Lyapunov exponent 0.1683 , and we observed that it decays to limit cycle exponentially with the largest Lyapunov exponent 0.0013 at the end of the transient phase of the obits.

\section{Conclusions}

(1) A periodically forced system called the generalized Ueda oscillator is presented. The damping term of this system is the product of a nonlinear function $\operatorname{sgn}(\mathrm{x})$ and an absolute function with a variant power.
(2) By replacing the signum function with continuous hyperbolic tangent function, the largest Lyapunov exponent of this system is calculated correctly, which shows that it has wide chaotic range.

(3) This system has complex bifurcations, including period-doubling bifurcation, interior crisis, pitchfork bifurcation, tangent bifurcation, and multiple attractors bifurcation. Multiple coexisting attractors are displayed by calculating the mean square deviations of the attractor from the reference.

(4) The complex hysteresis and chaotic transient phenomenon are observed, which shows that this system has rich dynamical behaviors.

The additional features of this system in terms of synchronization control, circuit implementation, and its application to secure communication deserve further study.

\section{Conflict of Interests}

The authors declare that they have no conflict of interests related to this study.

\section{Acknowledgments}

This work was supported by the National Nature Science Foundation of China (Grant nos. 61161006 and 61073187) and the SRF for ROCS, SEM. The authors are grateful for discussions with Professor Sprott J. C.

\section{References}

[1] van der Pol, "On relaxation-oscillations," The London, Edinburgh, and Dublin Philosophical Magazine and Journal of Science Series 7, vol. 2, pp. 978-992, 1926.

[2] G. Birkhoff and G.-C. Rota, Ordinary Differential Equations, John Wiley \& Sons, New York, NY, USA, 3rd edition, 1978.

[3] Duffing, Erzwungene Schwingungen Bei Veranderlicher Eigenfrequenz, Vieweg, Braunschweig, Germany, 1918.

[4] Y. Ueda, "Randomly transitional phenomena in the system governed by Duffing's equation," Journal of Statistical Physics, vol. 20, no. 2, pp. 181-196, 1979. 
[5] Moon and Holmes, The Road to Chaos, Aerial Press, Santa Cruz, Calif, USA, 1979.

[6] C. Bonatto, J. A. C. Gallas, and Y. Ueda, "Chaotic phase similarities and recurrences in a damped-driven Duffing oscillator," Physical Review E, vol. 77, Article ID 026217, 5 pages, 2008.

[7] C. Hayashi, Y. Ueda, N. Akamatsu, and H. Itakura, "On the behavior of self-oscillatory systems with external force," Transactions of the Institute of Electronics and Communication Engineers of Japan, vol. 53, p. 150, 1970.

[8] Y. Ueda, The Road to Chaos, Aerial Press, Santa Cruz, Calif, USA, 1992.

[9] C. Scheffczyk, U. Parlitz, T. Kurz, W. Knop, and W. Lauterborn, "Comparison of bifurcation structures of driven dissipative nonlinear oscillators," Physical Review A, vol. 43, no. 12, pp. 6495-6502, 1991.

[10] Q. Chen, Y. Hong, and G. Chen, "Chaotic behaviors and toroidal/spherical attractors generated by discontinuous dynamics," Physica A, vol. 371, no. 2, pp. 293-302, 2006.

[11] K. Murali, M. Lakshmanan, and L. O. Chua, "Bifurcation and chaos in the simplest dissipative non-autonomous circuit," International Journal of Bifurcation and Chaos, vol. 4, p. 1511, 1994.

[12] K. Srinivasan, "Multiple period doubling bifurcation route to chaos in periodically pulsed Murali-Lakshmanan-Chua (MLC) circuit," International Journal of Bifurcation and Chaos, vol. 18, no. 2, pp. 541-555, 2008.

[13] S. L. T. de Souza, I. L. Caldas, and R. L. Viana, "Damping control law for a chaotic impact oscillator," Chaos, Solitons and Fractals, vol. 32, no. 2, pp. 745-750, 2007.

[14] A. Ray, D. Ghosh, and A. Roy Chowdhury, "Topological study of multiple coexisting attractors in a nonlinear system," Journal of Physics A, vol. 42, no. 38, Article ID 385102, 2009.

[15] M. Dutta, H. E. Nusse, E. Ott, J. A. Yorke, and G. H. Yuan, "Multiple attractor bifurcations: a source of unpredictability in piecewise smooth systems," Physical Review Letters, vol. 83, no. 21, p. 4281, 1999.

[16] S. K. Baek and H.-T. Moon, “Complex hysteresis," Physics Letters A, vol. 352, no. 1-2, pp. 89-93, 2006.

[17] P. Berge, Y. Pomeau, and C. Vidal, Order within Chaos, Hermann, Paris, France, 1984.

[18] H. G. Li and G. Meng, "Nonlinear dynamics of a SDOF oscillator with Bouc-Wen hysteresis," Chaos, Solitions and Fractals, vol. 34, no. 2, pp. 337-343, 2007.

[19] J. M. T. Thompson, Nonlinear Mathematics and Its Applications, Cambridge University Press, Cambridge, UK, 1997, edited by P. J. Aston.

[20] H. P. W. Gottlieb and J. C. Sprott, "Simplest driven conservative chaotic oscillator," Physics Letters A, vol. 291, no. 6, pp. 385-388, 2001.

[21] Y. Ueda, "Randomly transitional phenomena in the system governed by Duffing's equation," Journal of Statistical Physics, vol. 20, no. 2, pp. 181-196, 1979.

[22] T. S. Parkerand and L. O. Chua, Numerical Algorithms for Chaotic Systems, Springer, 1989.

[23] A. Wolf, J. B. Swift, H. L. Swinney, and J. A. Vastano, "Determining Lyapunov exponents from a time series," Physica D, vol. 16, no. 3, pp. 285-317, 1985.

[24] W. J. Grantham and B. Lee, "A chaotic limit cycle paradox," Dynamics and Control, vol. 3, no. 2, pp. 159-173, 1993.

[25] R. F. Gans, "When is cutting chaotic?" Journal of Sound and Vibration, vol. 188, no. 1, pp. 75-83, 1995.
[26] K. Sun and J. C. Sprott, "Periodically forced chaotic system with signum nonlinearity," International Journal of Bifurcation and Chaos, vol. 20, no. 5, pp. 1499-1507, 2010.

[27] J. C. Sprott, "High-dimensional dynamics in the delayed Hénon map," Electronic Journal of Theoretical Physics, vol. 12, no. 3, pp. 19-35, 2006.

[28] J. A. Yorke and E. D. Yorke, "Metastable chaos: the transition to sustained chaotic behavior in the Lorenz model," Journal of Statistical Physics, vol. 21, no. 3, pp. 263-277, 1979.

[29] L. A. Zhu, A. Raghu, and Y. C. Lai, "Experimental observation of superpersistent chaotic transients," Physical Review Letters, vol. 86, pp. 4017-4020, 2001. 


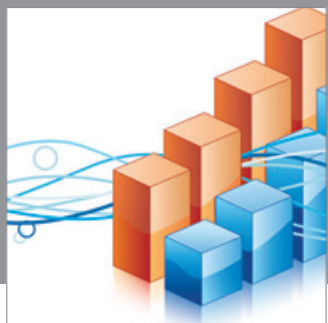

Advances in

Operations Research

mansans

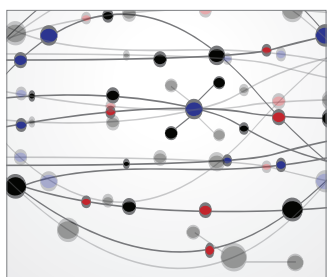

The Scientific World Journal
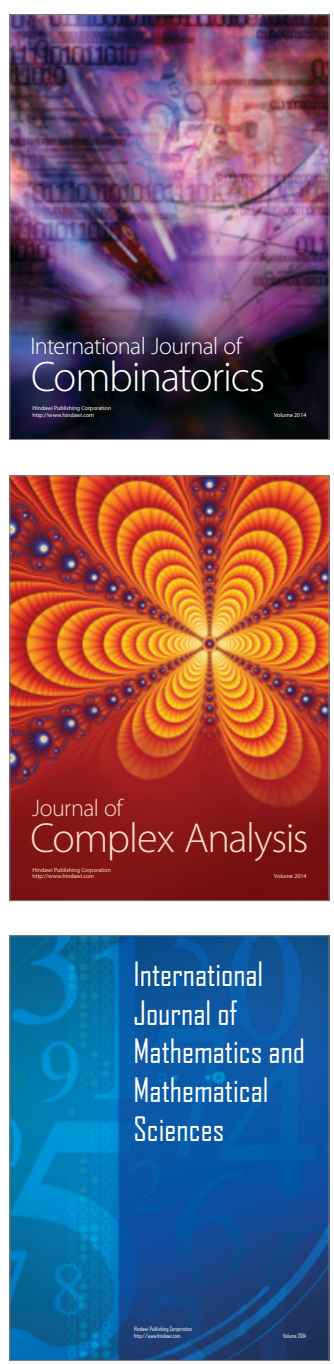
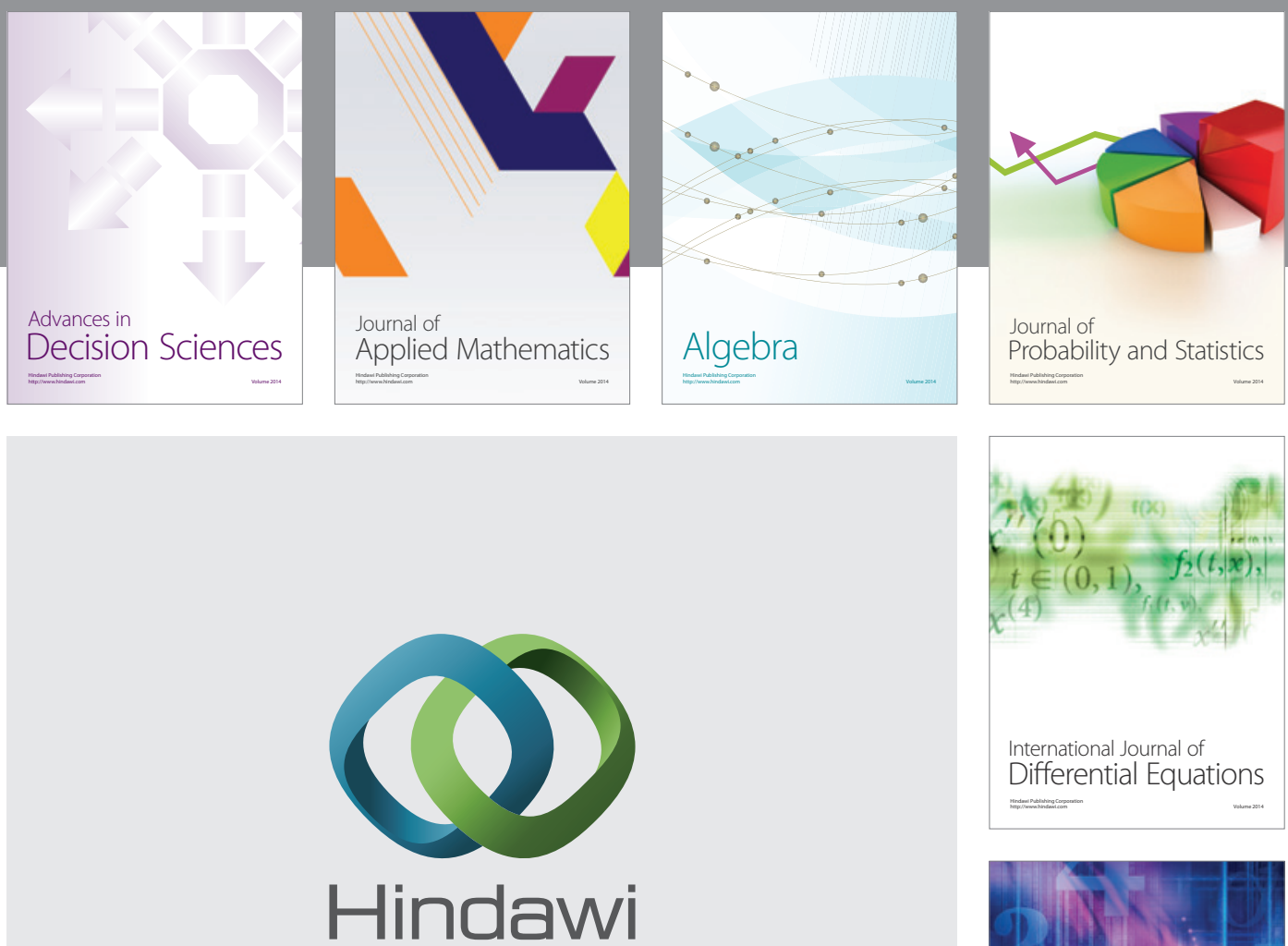

Submit your manuscripts at http://www.hindawi.com
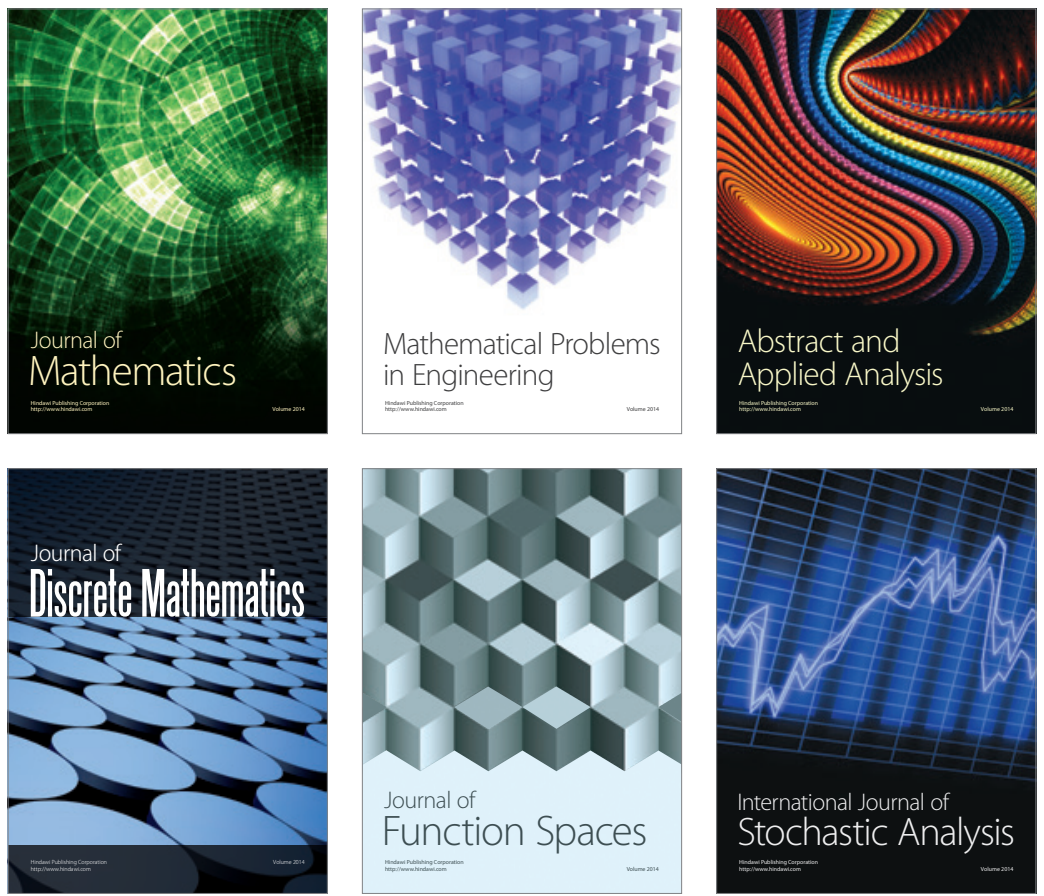

Journal of

Function Spaces

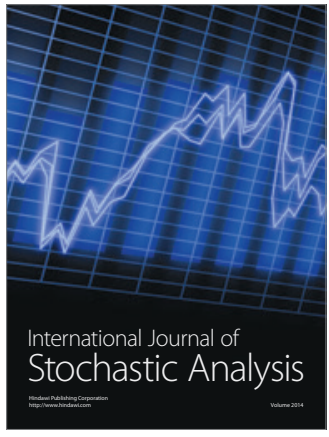

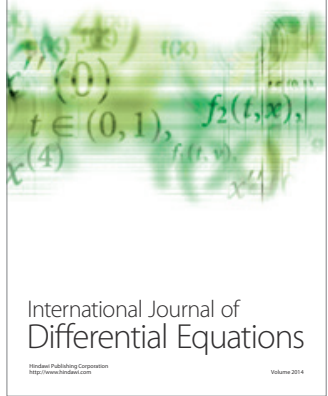
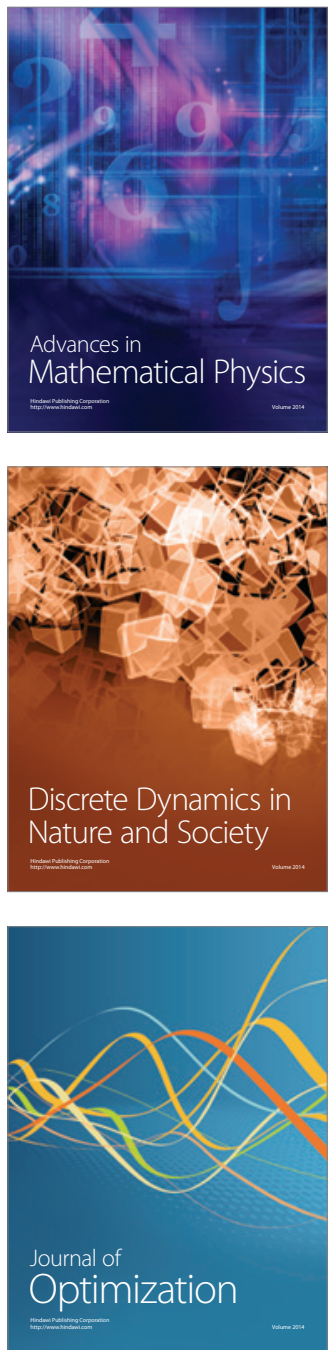